$16^{\text {th }}$ International Congress of Metrology, 08001 (2013)

DOI: $10.1051 /$ metrology/201308001

(c) Owned by the authors, published by EDP Sciences, 2013

\title{
Roughness and contamination characterizations of worn surfaces
}

\author{
Zaccaria Silvestri ${ }^{1, a}$, Shéhérazade Azouigui ${ }^{1}$, Patrick Pinot $^{1}$ and Mark Gee ${ }^{2}$ \\ ${ }^{1}$ Laboratoire commun de métrologie LNE-Cnam, 61 rue du Landy, 93210 La Plaine Saint-Denis, France \\ ${ }^{2}$ National Physical Laboratory NPL, Materials Division, Hampton Road, Teddington, TW11 0LW, UK
}

\begin{abstract}
The European project IND11 "Metrology to Assess the Durability and Function of Engineered Surfaces" (MADES), piloted by NPL, aims to increase the efficiency and utilization of engineered surfaces by studying wear phenomena. This is to increase the competitiveness of the industry, reducing costs on the one hand, and secondly the impact on the environment. In this context, the joint research laboratory (LNE-CNAM) characterizes the surface and contamination of different materials have undergone wear using an optical roughness-meter and a thermal desorption mass spectrometry device.
\end{abstract}

\section{Introduction}

The European project IND11 "Metrology to Assess the Durability and Function of Engineered Surfaces" (MADES) is aimed at providing improved metrology to assess engineered surfaces and facilitate the use of surface engineering. This should lead to major increases in the efficiency of motor vehicle transport, thereby reducing environmental impact. Overall, the work should increase the competitiveness of European industry. At present, the assessment of an engineered surface is currently largely empirical. Any step change improvements in performance will require the development of sound metrology for surface characterization.

The work of the project will provide the necessary innovative metrology to underpin and enable the development of future products across the European industry portfolio. The project is composed of eight work packages (WP) including Impact (WP 7) and Management (WP 8):

- Selection of materials and baseline testing (WP 1);

- Measurement of very small wear volumes (WP 2 and WP 4);

- Measurement of low friction (WP 4);

- Measurement of the temperature at wear interfaces (WP 3);

- Measurement of changes in chemistry at the contact interface between surfaces (WP 2 and WP 5);

- On-line measurement of wear and durability (WP 6);

- Assessment of the durability of tribological surfaces (WP 3).
The different partners of the project are: Alicona (Austria), BAM, FAU and PTB (Germany), CNAM (France), DTI (Denmark), INRIM (Italy), MIKES and VTT (Finland), and NPL (coordination, UK).

This paper will outline the objectives and motivations of the project and discuss specifically the work carried out by the CNAM group (Laboratoire commun de métrologie LNE-CNAM) on work packages 2 and 5.

\section{Assessment of wear}

\subsection{Presentation of the WP 2}

The work on the assessment of wear (WP 2) deals with the development of measurement methods on samples that have been subjected to wear so that the volume of wear can be determined. In particular in the task 2.2., CNAM (in collaboration with PTB) is in charge of the validation of an optical non-tactile roughness measurement technique for the characterization of damaged surfaces [1], [2], [3].

\subsection{Presentation of the optical roughness meter}

CNAM performs these measurements using its optical roughness-meter [4], [5]. This is based (see Figure 1) on the angular distribution of scattered laser light [6], [7], [8], whereby roughness (rms height and correlation length) is determined from the power spectral density (PSD) of the surface asperities.

\footnotetext{
${ }^{\mathrm{a}}$ Corresponding author: zaccaria.silvestri@cnam.fr
} 
The device mainly consists of a monochromatic ppolarized laser source at $635 \mathrm{~nm}$, an optical arrangement (collimation and filtering system), a motion-controlled sample holder, a detector on a movable arm and a photomultiplier tube.

Figure 1. Schematic of the light scattering geometry.

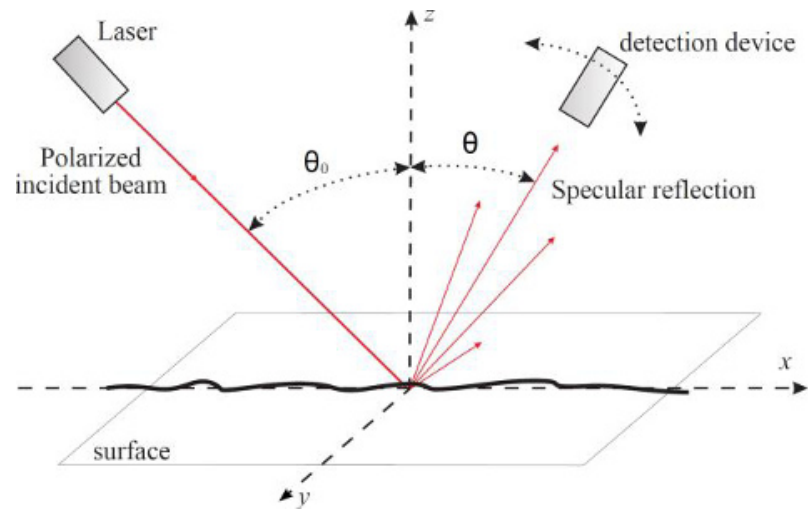

In a first step, in order to provide information about the homogeneity of the surface, a scattered light map for a given scattering angle is plotted. This consists in setting to a fixed value, translating the plate along the directions over a given area and measuring the scattered light intensity for each scanned site. Using this map, interesting sites are selected for further investigation by PSD measurements.

Compared to others methods, this device is relatively fast. Indeed, it takes about $3 \mathrm{~min}$ to measure the angular distribution of the scattered light for a given site on a sample and about $30 \mathrm{~min}$ for a scattered light map on 1 $\mathrm{mm}^{2}$ with steps of $25 \mu \mathrm{m}$.

\subsection{Characterization of wear tracks on $100 \mathrm{Cr} 6$ steel sample}

The surface studied in this work was made from hardened and tempered 100Cr6 steel (supplied by BAM) as bearing steel, and finished in a two-step finishing process. It is an uncoated plane disk (Figure 2.a) used in $\mathrm{SRV}^{\circledR}$ test equipment. It was subjected to a lubricated tribological test in a reciprocating sliding tribometer: a polished $100 \mathrm{Cr} 6 \mathrm{H}$ ball of $5 \mathrm{~mm}$ diameter oscillated against the disc during 150000 cycles under respectively 300 and $30 \mathrm{~N}$ loads at a $50 \mathrm{~Hz}$ frequency [3], resulting in resp. $2.6 \mathrm{~mm}$ by $1 \mathrm{~mm}$ and $5 \mathrm{~mm}$ by $0.3 \mathrm{~mm}$ wear tracks (Figure 2.b).
Figure 1. Photographs of the uncoated $100 \mathrm{Cr} 6 \mathrm{H}$ SRV ${ }^{\circledR}$ sample with 2 wear tracks: (a) Overall view; (b) Zoom of tracks T1 and $\mathrm{T} 2$.

(b)

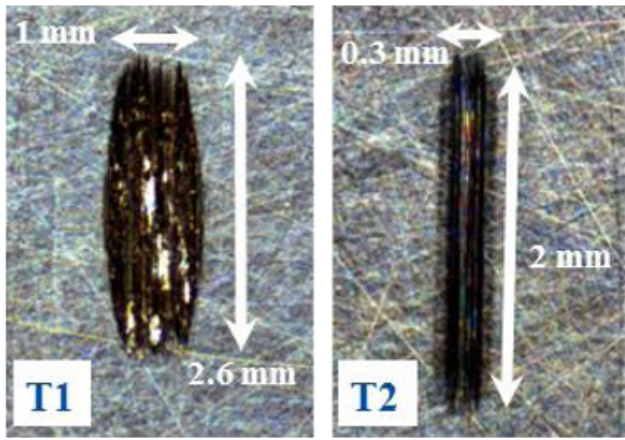

Figure 2 presents scattered light maps for both tracks T1 and $\mathrm{T} 2$, which allow one to distinguish between worn and unworn areas on the surface although it is not a topographical view of the surface profile.

Figure 2. Scattered light maps for $\theta=30^{\circ}$. (a) $3 \mathrm{D}$ view. (b) iso view.

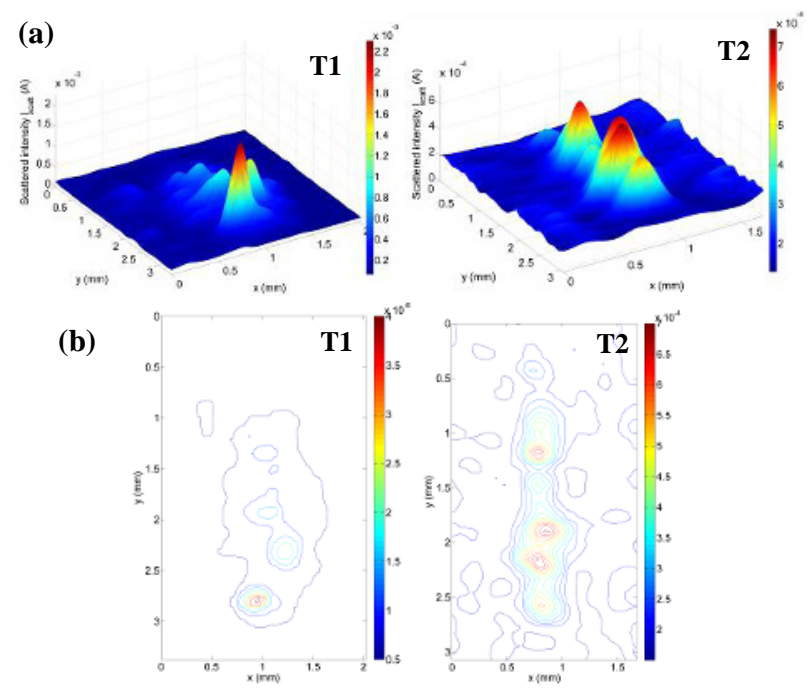

Figure 3 presents PSD curves, also called "roughness spectra" from unworn (i.e outside) and worn (i.e inside $\mathrm{T} 1$ and $\mathrm{T} 2$ tracks) areas. 
Figure 3. PSD S(f) outside tracks, inside T1 and inside T2.

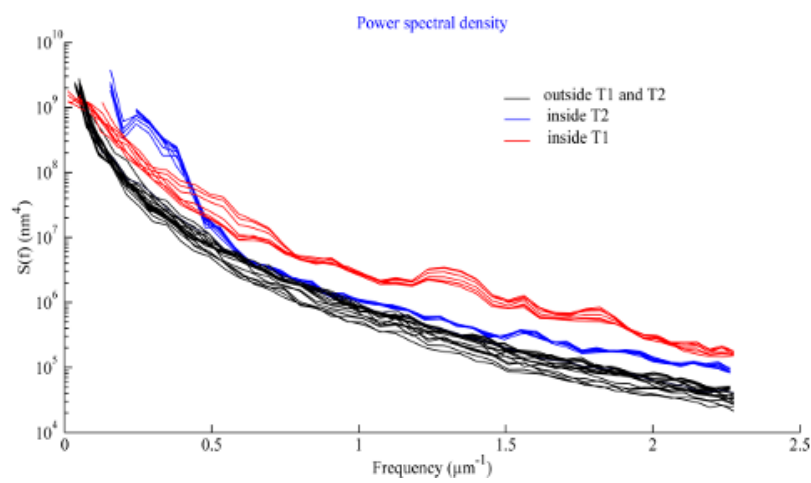

In these roughness spectra, worn and unworn area are clearly identified for the all spatial frequencies available on optical measurements.

\section{Measurement of interface chemistry}

\subsection{Presentation of the WP 5}

The work on the measurement of interface chemistry (WP5) is aimed at the development of methods to characterize the chemical changes that have occurred at the worn surface on the scale of a few millimeters. Two methods are being compared: Raman spectroscopy (BAM, NPL), and Thermal Desorption mass Spectrometry (TDS) by CNAM. Samples have been assessed using a TDS device on worn and unworn surfaces.

\subsection{Presentation of TDS apparatus}

The basic principle is to induce desorption of physisorbed molecules on a surface by heating it under low pressure. By performing this thermodesorption under vacuum, it is simple to analyze desorbed molecules using a mass spectrometer. Temperature Programmed Desorption (TPD) is used because of its easiest implementation: a regulated temperature ramp allows to follow the partial pressure (or intensity) of desorbed molecules and determine kinetic and thermodynamic parameters during a desorption [9].

The experimental device [10][11] (Figure 4) is mainly composed of three main parts: an analysis chamber high resolution quadruple mass spectrometer, a load-lock system and a glove-box for the transfer of sample under inert gas.
Figure 4. Photograph of the Thermal Desorption mass Spectrometer (TDS).

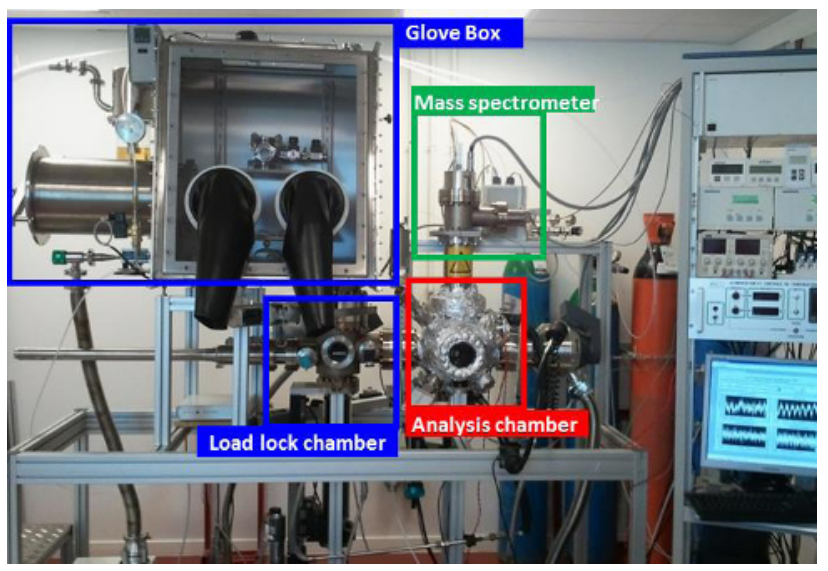

\subsection{Example of results}

In the same way as presented in WP 2 section, a 100Cr6 sample ref. cd-401 (Figure 5.a) has been run against a polished $100 \mathrm{Cr} 6 \mathrm{H}$ ball during 150000 cycles under $300 \mathrm{~N}$ at a $50 \mathrm{~Hz}$ frequency. In order to be able to detect contamination, height wear tracks have been processed on a sample (Figure 5.b). Another sample without track is used as reference.

Figure 5. Photographs (from BAM) of the uncoated $100 \mathrm{Cr} 6 \mathrm{H}$ $\mathrm{SRV}^{\circledR}$ sample with 8 wear tracks: (a) overall view; (b) zoom on each track.

(a)

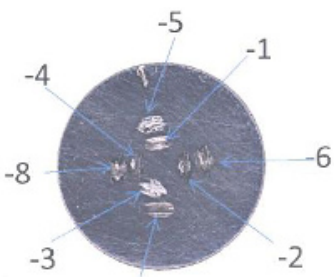

(b)
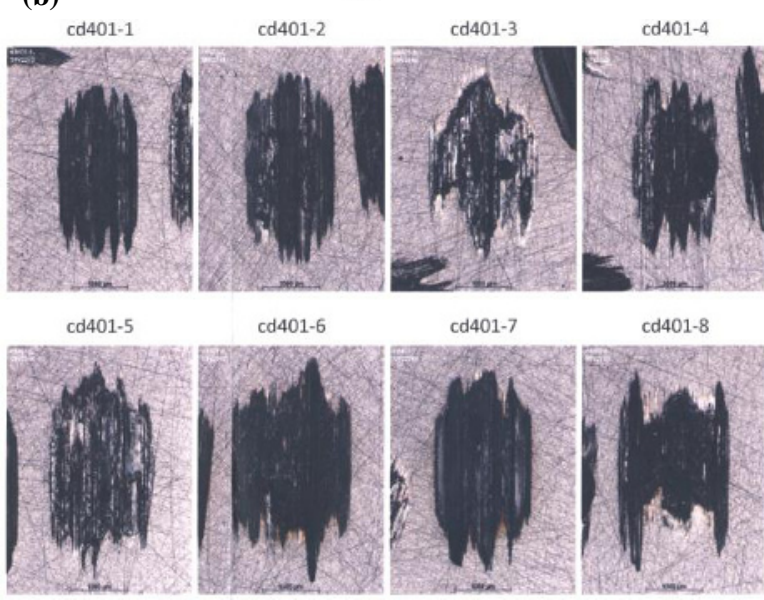
Before the study, samples have been cleaned by a thermal desorption in vacuum at $600^{\circ} \mathrm{C}$ during 2 hours in order to desorb oil used during tribological tests. Then after a twoweek exposition to air lab, reference and damaged samples have been successively analyzed by thermal desorption using this same optimized experimental protocol:

- Ramp temperature: $15^{\circ} \mathrm{C} / \mathrm{min}$;

- Maximum temperature: $600{ }^{\circ} \mathrm{C}$;

- Time of thermal desorption: 2 hours.

Figure 6 presents differential mass spectra for different surface temperatures for the main detected atomic masses. The main contaminations are water $\left(\mathrm{H}_{2} \mathrm{O}\right)$, dioxide carbon $\left(\mathrm{CO}_{2}\right)$ and to a lesser degree hydrocarboneous compounds.

Figure 6. Differential mass spectra between Reference and Cd-401 samples: $\left(I_{\mathrm{ref}}-I_{\mathrm{cd}-401}\right)=\mathrm{f}(\mathrm{m} / \mathrm{z})$. Mass spectra: (a) from 11 to $16 \mathrm{u}$. (b) from 37 to $48 \mathrm{u}$ and (c) from 50 to $120 \mathrm{u}$.

(a)

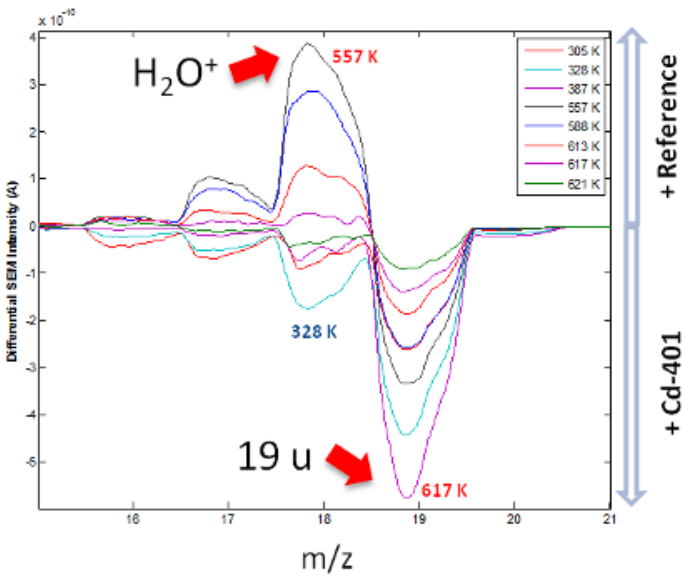

(b)

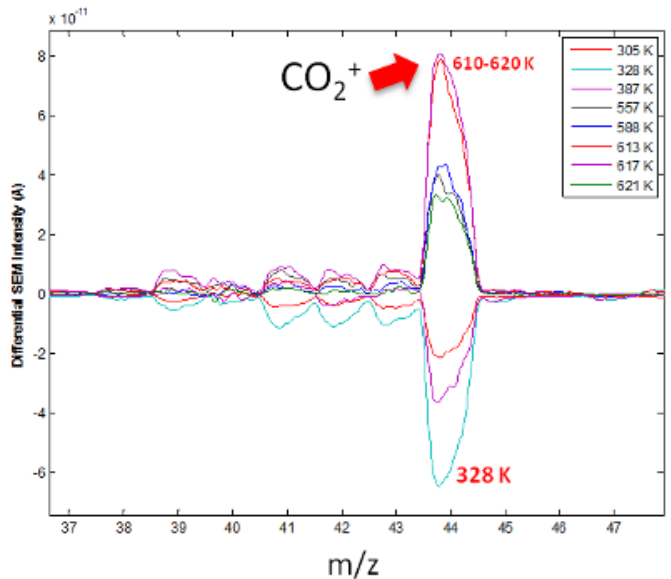

(c)

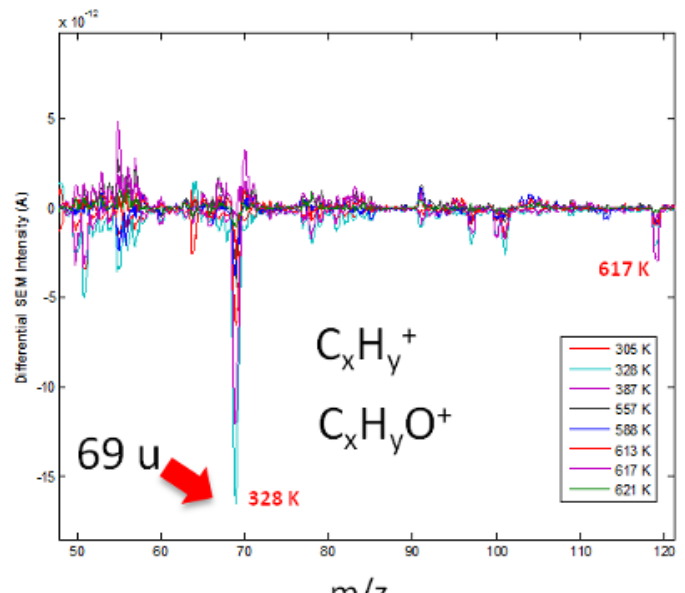

For temperatures between 300 and $450 \mathrm{~K}, \mathrm{H}_{2} \mathrm{O}$ and $\mathrm{CO}_{2}$ more desorb on the damaged sample. From $450 \mathrm{~K}$, it is on the contrary the reference sample which desorbs mores. For temperatures between 300 et $630 \mathrm{~K}$, masses $19 \mathrm{u}, 69 \mathrm{u}$ (possible fragments of $\mathrm{C}_{\mathrm{x}} \mathrm{H}_{\mathrm{y}}{ }^{+}$or $\mathrm{C}_{\mathrm{x}} \mathrm{H}_{\mathrm{y}} \mathrm{O}^{+}$) and $119 \mathrm{u}$ are also more desorbing on the damaged sample.

\section{Conclusion}

For WP 2, using an optical roughness-meter, worn and unworn areas of a 100Cr6 sample have been characterized. Clear different between these areas have been observed in terms of roughness. However, to understand better wear processes, further studies are in progress using a sample with a given contact ball force and different cycles (50 000 to 200000 ) of wear and a sample with a given cycle and different contact force $(10 \mathrm{~N}$ to $50 \mathrm{~N})$.

For WP 5 and especially for contamination analysis on worn area using TDS device, an optimized experimental protocol (choice of ramp temperature, choice of the maximum temperature...) has been optimized. Results would appear to show few differences between worn and unworn areas in terms of contamination especially for the case of water. The influence of the environment such as the effect of humidity, and the effect of cleaning on the chemical species at the wear contact will also be evaluated. An optimized measurement procedure will be developed with quantified measurement uncertainties and that is validated by comparison to other measurement methods.

\section{Acknowledgments}

This work is part of the EURAMET Joint Research Project MADES. It received funding from the European Union's Seventh Framework Programme, ERANET Plus, under grant agreement 217257. The authors thank the partners of the MADES project and are grateful to Dirk 
Spaltmann, Manfred Hartelt, Christian Scholz from BAM and Harald Bosse from PTB.

\section{References}

1. B. Bhushan, New York, John Wiley \& Sons, (2002).

2. C. Scholz, D. Spaltmann, and M. Woydt, Wear, 270, 7-8, pp. 506-514, (2011).

3. D. Klaffke and M. Hartelt, Tribol. Lett., 1, 4, pp. 265-276, (1995).

4. C. Zerrouki, F. Miserey, and P. Pinot, Eur Phys Ap, 1, pp. 253-259, 1998.

5. Z. Silvestri, C. Zerrouki, and P. Pinot, Rugosité d'un étalon de masse: évaluation de l'incertitude par une méthode de Monte Carlo, Congrès international de métrologie, Paris, France, (2009).

6. J. M. Bennett and L. Mattsson, Introduction to surface roughness and scattering. Optical Society of America, Washington, D.C., (1989).

7. J. M. Elson and J. M. Bennet, J Opt Soc Am, 69, pp. 31-67, 1979.

8. J. C. Stover, Optical scattering: measurement and analysis, 3rd ed. Bellingham, Wash: SPIE Press, (2012).

9. Y. Amenomiya and R. J. Cvetanovic, J. Physic Chem., 67, 1, pp. 144-147, 1963.

10. Z. Silvestri, D. du Colombier, S. Macé, and P. Pinot, Rev. Française Métrologie, 23, pp. 11-20, (2011).

11. Z. Silvestri and P. Pinot, Thermal Desorption mass Spectrometry (TDS). Application on mass metrology, XIX IMEKO World Congress Fundamental and Applied Metrology, Lisboa, Portugal, (2009). 